\title{
An integrated approach for early forest fire detection and verification using optical smoke, gas and microwave sensors
}

\author{
N. von Wahl ${ }^{1}$, S. Heinen ${ }^{1}$, H. Essen ${ }^{1}$, W. Kruell ${ }^{2}$, \\ R. Tobera ${ }^{2} \&$ I. Willms ${ }^{2}$ \\ ${ }^{I}$ Fraunhofer Institute for High Frequency Physics and Radar Techniques \\ FHR, Germany \\ ${ }^{2}$ Department of Communication Systems (NTS), \\ University of Duisburg-Essen, Germany
}

\begin{abstract}
In 2008 the research project "International Forest Fire Fighting" (iWBB) was funded by the Minister for Economic Affairs and Energy of the State of North Rhine-Westphalia, Germany. A group of companies, research institutes and universities are working together to develop an integrated, but modular system. An integrated approach for early forest fire detection and suppression is based on an adequate combination of different detection systems depending on wildfire risk, the size of the area and human presence affiliated with an adequate logistical infrastructure, training by simulation, and innovative extinguishing technology based on armoured tracked fire fighting vehicles.

As large areas have to be monitored, only remote sensing technologies (e.g. video based systems) are able to perform early detection adequately. To reduce false alarms a remote controlled unmanned aerial vehicle (UAV) equipped with gas sensors and a thermal camera flies to a potential fire to specify the origin of the reported cloud. The UAV can also be used as a scout for fire fighters. After successful fire extinction an unmanned blimp can be used as a fireguard to reduce the risk of reignition of the fire. As monitoring tools, a microwave radiometer, which is capable of detecting hot spots even under insufficient vision (due to smoke clouds and below the ground surface), gas and smoke sensors and a thermal camera are mounted on the blimp. The benefit of a blimp is a higher payload.
\end{abstract}


This paper presents an investigation of an early forest fire detection system on the basis of indoor (performed in the fire lab of the University of DuisburgEssen) and outdoor tests. A commercial highly sensitive aspirating smoke detector, two gas sensors $\left(\mathrm{H}_{2}\right.$ and $\left.\mathrm{C}_{\mathrm{X}} \mathrm{H}_{\mathrm{X}}\right)$ and the microwave radiometer are detailed and detection algorithms are described. A general overview about the project and the carrier platforms is presented.

Keywords: fire detection, gas sensors, microwave radiometer, smoke detector, $U A V$, remote sensing, fire scout, fireguard.

\section{General outline}

Fast and effective detection is a key factor in forest fire fighting. To avoid uncontrollable wide spreading of forest fires it is necessary to detect fires in an early state and to prevent the propagation. It is important to move adequate fire equipment and qualified operational manpower as fast as possible to the source of the fire. Furthermore, an adequate logistical infrastructure for sufficient supply with extinguishing devices and maintenance is necessary, as well as continuous monitoring of fire spread. Moreover, the training of personnel is an important component for successful combating of forest fires. An integrated approach for forest fire detection and suppression is based on a combination of different detection systems depending on wildfire risk, the size of the area and human presence, consisting of all necessary parts, such as early detection, remote sensing techniques, logistics, training by simulation, and fire-fighting vehicles, see fig. 1.

Different risk levels, the size of the area and human presence define the applied sensing techniques. Small high risk areas can be observed by local staff. For very large and low risk areas satellite and aero monitoring is possible.

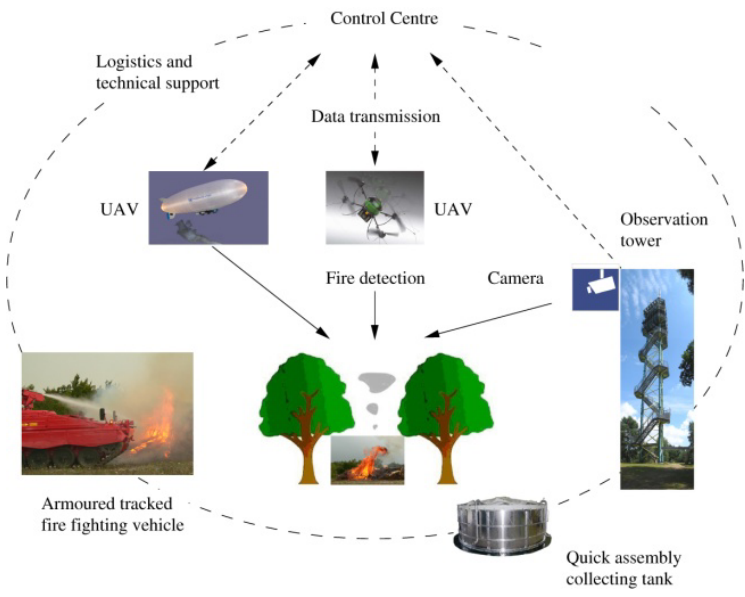

Figure 1: Schematic structure of the integrated forest fire detection and fire fighting system. 

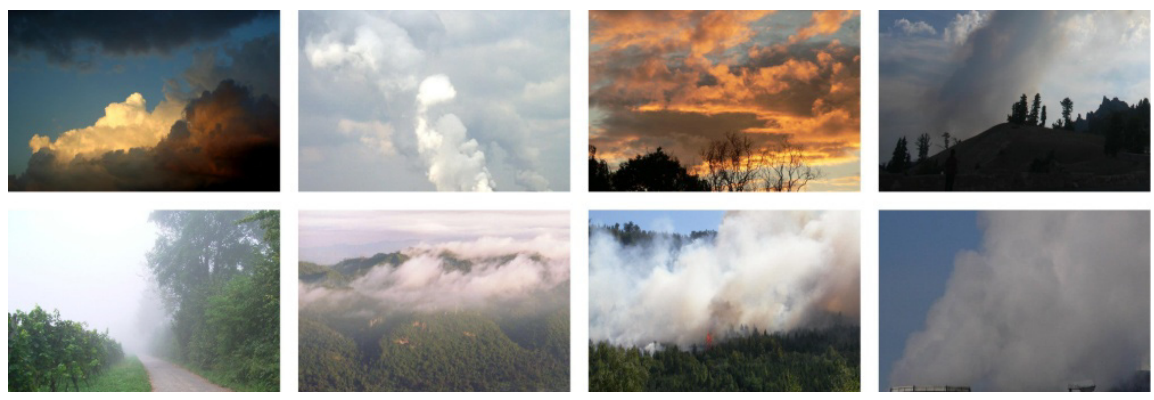

Figure 2: $\quad$ Scenes with alarm and false alarm situations.

Especially in the eastern part of Germany, several hundred observation towers equipped with camera-based systems have been built to observe forests. Recorded video sequences are transmitted to a control centre and analyzed by appropriate software. If a fire is clearly identified, fire suppression will be initialized by an alarm going directly to the fire brigade.

As known from other fire detection technologies the problem of false alarms requires additional measures for alarm verification. Possible false alarms are caused e.g. by dust produced by farmers, pollen, fog, smoke and water plumes produced by power plants, fig. 2. In case of an ambiguous decision a remote controlled Unmanned Aerial Vehicle (UAV) equipped with gas sensors and an infrared camera can fly to the place where a fire is assumed to provide detailed pictures and a multitude of other measured data. Depending on these data a fire will be confirmed or unconfirmed.

If the source of a fire is not accessible for common fire fighting vehicles with conventional tyres it is only possible to extinguish the fire from the air or by socalled "smoke jumpers". A new solution is a reconstructed armoured and tracked fire fighting vehicle as known from military use, supporting existing airborne fire fighting ground-based right at the source of the fire in tough, rugged terrain. An innovative extinguishing system with high-pressure vortex technology was developed to provide a vehicle with extremely low water consumption [1].

\section{Carrier platforms for the detection system}

A very high reliability of fire detection and a concomitant low false alarm rate can be achieved by the combination of an infrared camera, a microwave radiometer and additional sensors of fire products respectively smoke particles, working with conventional and established technologies of fire detection. These detectors should be insensitive to dust and water particles. Depending on the application a selection of these sensors will be mounted on a mini-drone or a blimp. For both platforms, available space and weight are limited.

\subsection{Early forest fire verification with a mini-drone}

Carrier platform for the detection system in case of early fire detection is an autonomously flying video drone with excellent navigation skills in every 
territory. The AirRobot AR100-B (fig. 3a) offers a budget priced alternative to the normal aerial surveillance by a manned helicopter. The UAV enables the fire brigade to have maximum awareness of the situation and the occurrences during their mission. It can either be used for confirmation of an alarm detected by a video system or as well as a scout, helping to find hot spots especially at night while no fire fighting planes are flying. The UAV has a diameter of one meter, a weight of about one kilogram and a silent electric drive (4 brushless- and gearless DC-motors). It can be flown without any pilot experience. Data transmission and control in real-time is possible by RF devices. The telemetrydata is shown live on the ground station and can be tracked on a map in realtime. The whole processing for obstacle detection and collision avoidance is taking place autonomously in the AirRobot.

\subsection{Hot spot surveillance with a blimp}

In the case of monitoring extinguished fires, observation with a radiometer, gas and smoke detectors and a thermal camera attached to a blimp (fig.3b) is

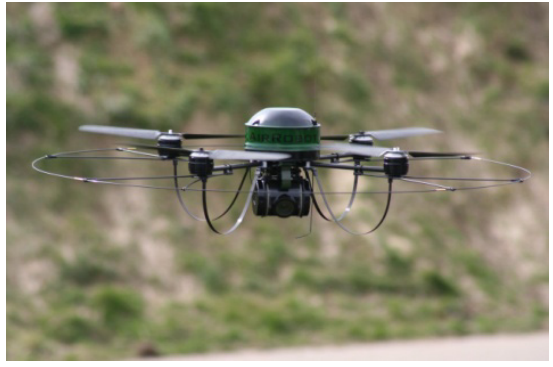

(a)

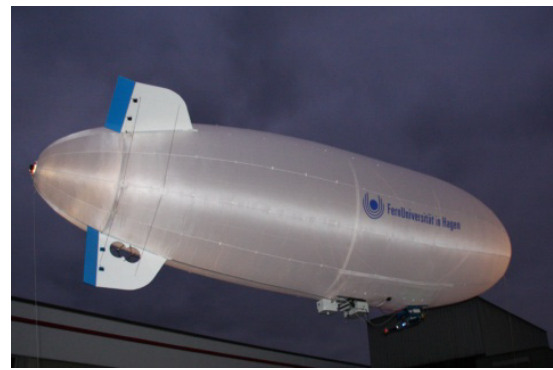

(b)

Figure 3: (a) AirRobot UAV. (b) Blimp as a fireguard.

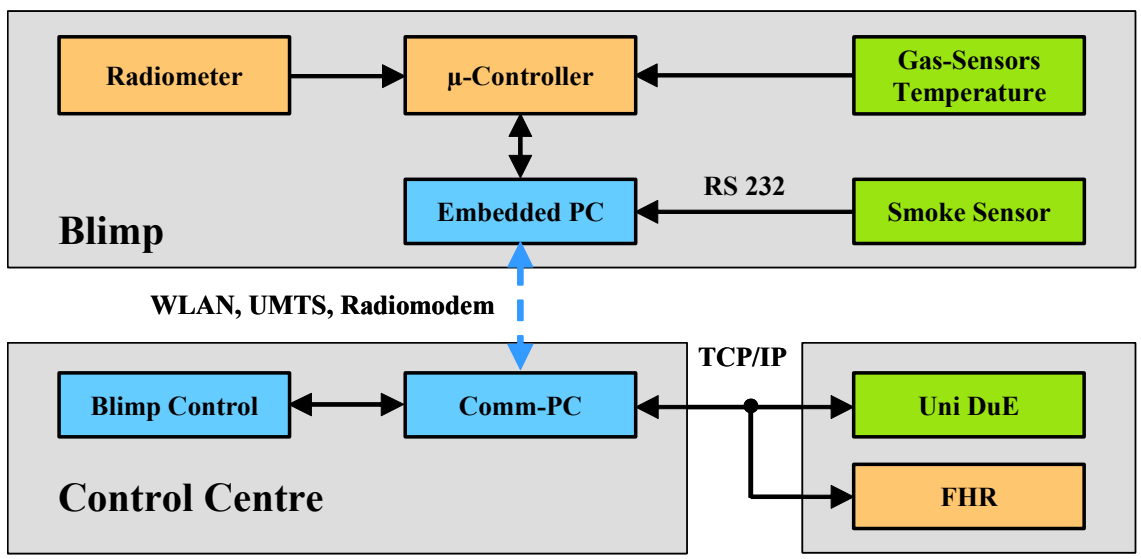

Figure 4: Communication structure for the blimp. 
performed. Benefit of the 9 meter long airship with $2.3 \mathrm{~m}$ diameter is a high payload of $7 \mathrm{~kg}$ including batteries. The embedded-PC shown in fig. 4 controls the blimp as well as the communication and data transfer to the ground station.

The ground station consists of a user interface (Comm-PC) to transmit and receive flight information from the blimp via WLAN. Users (Uni-DuE, FHR) have to request their sensor data via TCP/IP at the ground station. The received data are combined with GPS data of the blimp and a time stamp for visualization and fed into the detection algorithms, determining whether an alarm is executed or not.

\section{Early fire detection with gas sensors and smoke detectors}

The sensor system shall be used to verify an ambiguous situation detected by a video-based system as well as observing an extinguished fire. Therefore sensors have to be widely immune against disturbances like steam, fog, dust pollution and condensing water, which cause video-based systems to give false alarms.

If fire gases are carried to the detector by the airflow, they are analysed with different semiconductor gas sensors. A gas-permeable protective cap made of sintered metal protects the sensor elements against soiling with dust and humidity. Conditional on its principle the sensor array is not affected by nuisance aerosols like dust, dirt, mist or condensing water [3].

Early forest fire detection sensors have to fulfil a lot of specific requirements compared to conventional applications. High sensitivity is needed to detect even low smoke concentration; dilution and extreme turbulence caused by wind are essential factors. Due to high occurrence of hydrogen during an open fire $\mathrm{a}_{2}$ Sensor [0 - 10ppm] was implemented $[4,5]$. Main features of this semiconductor gas sensor (GTE GSME) are a very fast response time and a high sensitivity [3]. A $\mathrm{C}_{\mathrm{X}} \mathrm{H}_{\mathrm{X}}$-Sensor $[0-5 \mathrm{ppm}]$ is used because hydrocarbon sensors are sensitive to organic fire products. Fast temperature fluctuations are measured by a temperature sensor. For smoke detection a high sensitive aspirating system (Hekatron ASD535 [6]) is used to detect even low smoke concentrations. The structure of the implemented detection system is shown in fig. 5 .

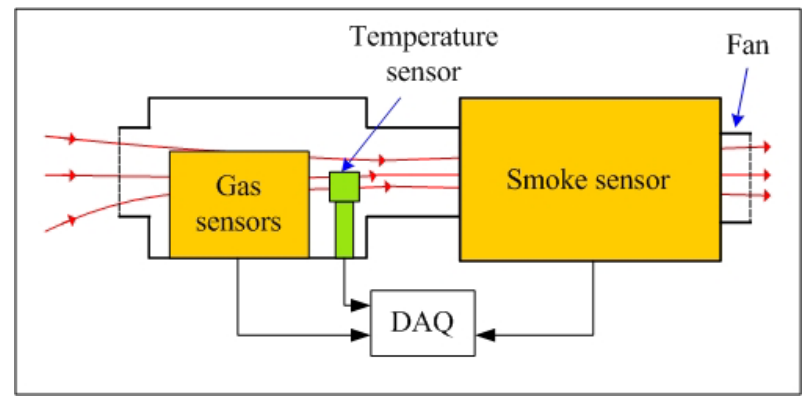

Figure 5: $\quad$ Structure of the sensor system and the internal airflow. 


\section{Detection algorithm for gas and smoke detection}

With the analysis of sensor data measured by the AirRobot drone a pre-alarm of a video-based system shall be confirmed or unconfirmed. The measured sensor signals for hydrogen $\left(\mathrm{N}_{\mathrm{H} 2}\right)$, hydrocarbon $\left(\mathrm{N}_{\mathrm{CH}}\right)$ concentration and temperature $\left(\mathrm{N}_{\mathrm{T}}\right)$ are transmitted to the control centre and fed into the detection algorithm, according to eqn. $(1,2)$. The decision "alarm / no alarm" is the result of the output value $\mathrm{S}_{\mathrm{gas}, \mathrm{T}}$ compared to the alarm threshold. Due to the application and the limited payload of the UAV a smoke detector is not implemented.

The observation of extinguished areas is performed by a blimp in order to detect buried hotspots and reignited seats of fire. This application requires an additional smoke detector and the detection algorithm has to be enlarged, according to eqn. $(3,4)$. The measured sensor signals for smoke density $\left(\mathrm{N}_{\mathrm{D}}\right)$, hydrogen $\left(\mathrm{N}_{\mathrm{H} 2}\right)$, hydrocarbon $\left(\mathrm{N}_{\mathrm{CH}}\right)$ and temperature $\left(\mathrm{N}_{\mathrm{T}}\right)$ are transmitted from the blimp to the control centre. Fig. 6 shows the structure of the implemented detection algorithm.

$$
\begin{gathered}
\mathrm{S}_{\mathrm{gas}, \mathrm{T}}=\left[\left(\mathrm{N}_{\mathrm{H} 2 \text {,filtered }} \mathrm{K}_{\mathrm{H} 2}+\mathrm{N}_{\mathrm{CH}, \text { filtered }} \mathrm{K}_{\mathrm{CH}}\right)\left(1+\mathrm{N}_{\mathrm{T}, \text { filtered }} \mathrm{K}_{\mathrm{T}}\right)\right] \\
\mathrm{S}_{\mathrm{gas}, \mathrm{T}} \geq \text { Alarm threshold } \Rightarrow \text { Alarm }
\end{gathered}
$$

The algorithm can be adapted to the environmental conditions by subtraction of the sensors' quiescent values; just variations will cause an alarm. Pulse forming is necessary because of different response characteristics of the sensors. Especially in situations with high turbulences with very short smoke and gas pulses it is helpful. The third part of the algorithm is weighting and fusion of the pre-processed sensor signals.

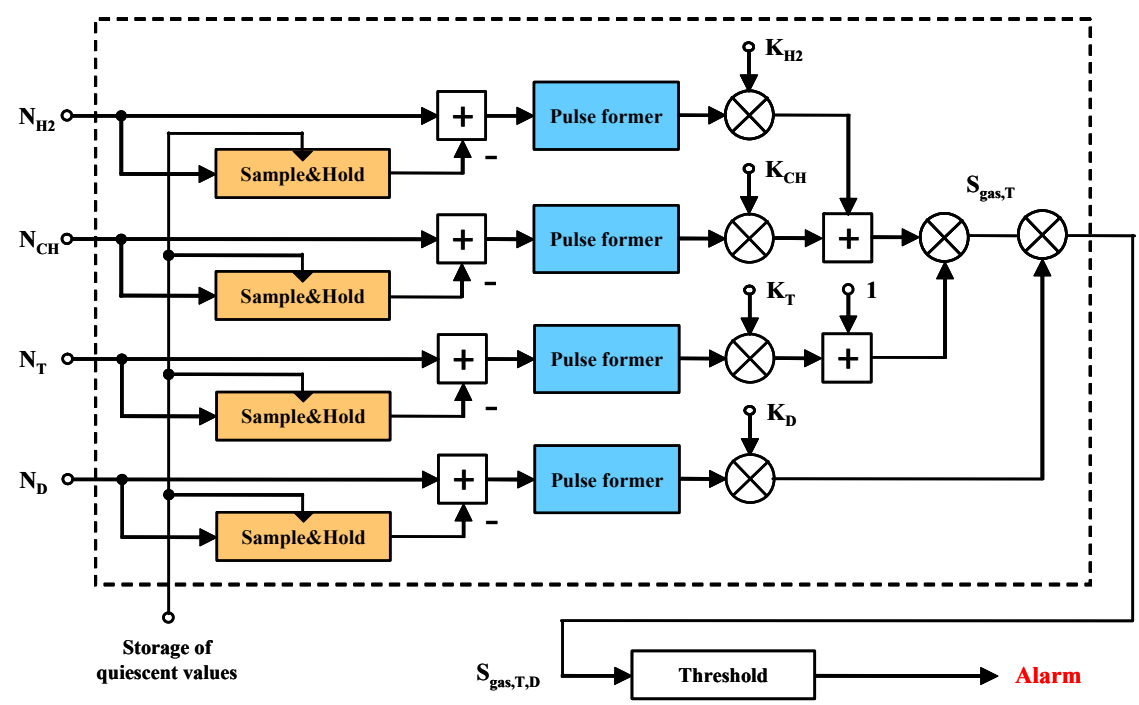

Figure 6: Structure of the algorithm. 


$$
\begin{gathered}
S_{\text {gas }, T, D}=S_{\text {gas, } T}\left(N_{D, \text { filtered }} K_{D}\right) \\
S_{\text {gas,T,D }} \geq \text { Alarm threshold } \Rightarrow \text { Alarm }
\end{gathered}
$$

The gas production of open and smouldering fires is different. Due to the chosen weight $\left(\mathrm{K}_{\mathrm{H} 2}\right.$ und $\left.\mathrm{K}_{\mathrm{CH}}\right)$ of the gas sensor signals the susceptibility of the algorithm to both types of fires is similar. The algorithm also includes the temperature and the light scattering measurement.

\section{Microwave radiometer}

The proposed microwave radiometer detects fire radiation at $22.3 \mathrm{GHz}$. According to Planck's law a black body reaches its maximum of the radiation power in the infrared region. With the assumption that fire has similar characteristics as a black body, the wide use of conventional IR-cameras in fire detection applications is justified. In [7] it was shown that fire detection is also possible with microwave sensors. Additionally, good transmission behaviour in smoke, dust and fog is characteristic for sensors working in the lower $\mathrm{GHz}$ region [8]. There are long wavelengths in the background, which lead to less absorption and scattering in particles of the materials compared to infrared light. This characteristic of microwaves can be exploited for hot spot detection in smoky environments. Furthermore, after extinction of a forest fire, hot spots often occur and propagate under the upper surface of the earth, which often causes reignition of forest fires. A microwave radiometer allows detection of hot spots even if covered by thin layers of leaves, scrub and the like.

Fig. 7 presents the principle of the radiometer. Via a switch a signal is alternately received from the scene of interest (through a planar group antenna) and a noise source. This can be exploited for signal normalisation. In the

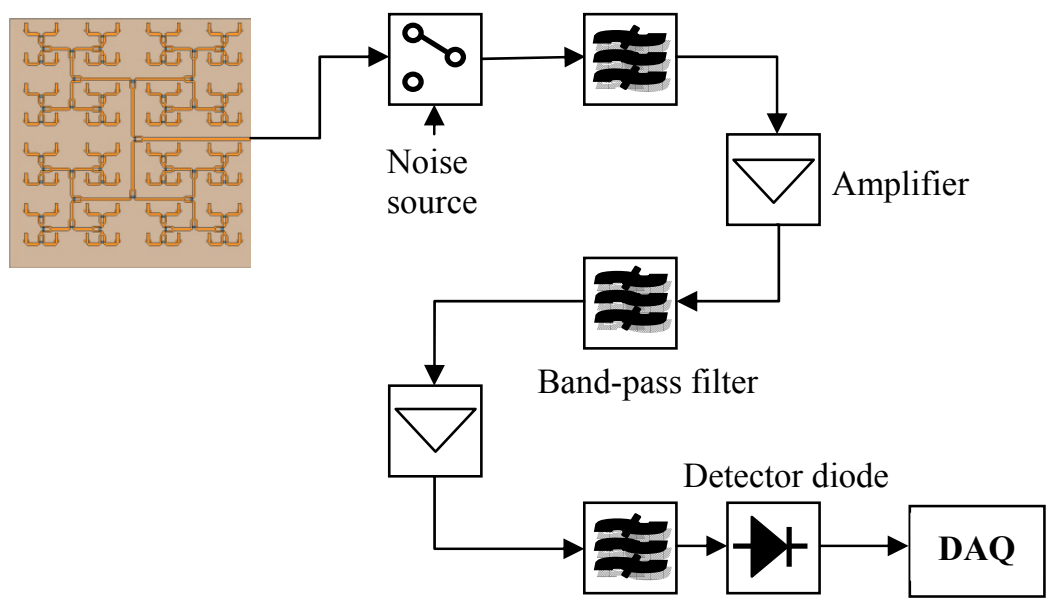

Figure 7: Block diagram of the microwave radiometer. 
following processing the signal gets amplified and filtered. To prevent disturbances of the radiometric signal by other systems, the frequency of the radiometer was adapted to a radio-astronomical band with a bandwidth of $600 \mathrm{MHz}$. For data processing in the DAQ the signal must be converted from power into voltage using a detector diode.

The image resolution of the radiometer is dependent on the antenna opening angle and the flying altitude and follows basic trigonometric rules. The antenna opening angle can be calculated from frequency $f$ and antenna size $d$, eqn. (5), $c_{0}$ being the speed of light in a vacuum.

$$
\alpha=72 * \frac{c_{0} / f}{d}
$$

For the frequency of $22.3 \mathrm{GHz}$, a flying altitude of $30 \mathrm{~m}$, an antenna size of $20 \mathrm{~cm}$ and an opening angle of approx. $5^{\circ}$, radiation can be detected from a square cell of $2.6 \mathrm{~m}$ edge length, the so called footprint of the system. The detection probability of a fire is highest if the footprint overlaps the fire completely. With the radiometer described above, only one pixel is generated. To produce several pixels, more than one antenna, a scanning system or a systematic flying platform must be implemented.

Fig. 8 shows the radiometer together with a temperature sensor, processing boards (PCB) and DAQ. The size is $105 \mathrm{~mm} \times 150 \mathrm{~mm} \times 73 \mathrm{~mm}$ and the weight including the case $(300 \mathrm{~g})$ is approximately $800 \mathrm{~g}$. Further reductions are possible by designing chip based high frequency components and merging all single boards.

To increase the field of view of the radiometer the planar group antenna is attached to a small video camera with vertical and horizontal scan axis, fig. 9. Both sensors get data from the same direction. These data sets can be superimposed.

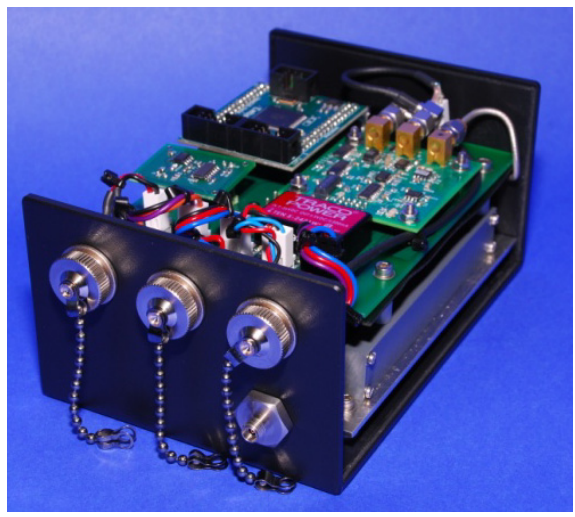

Figure 8: $\quad$ Image of the $22.3 \mathrm{GHz}$ radiometer without antenna. 

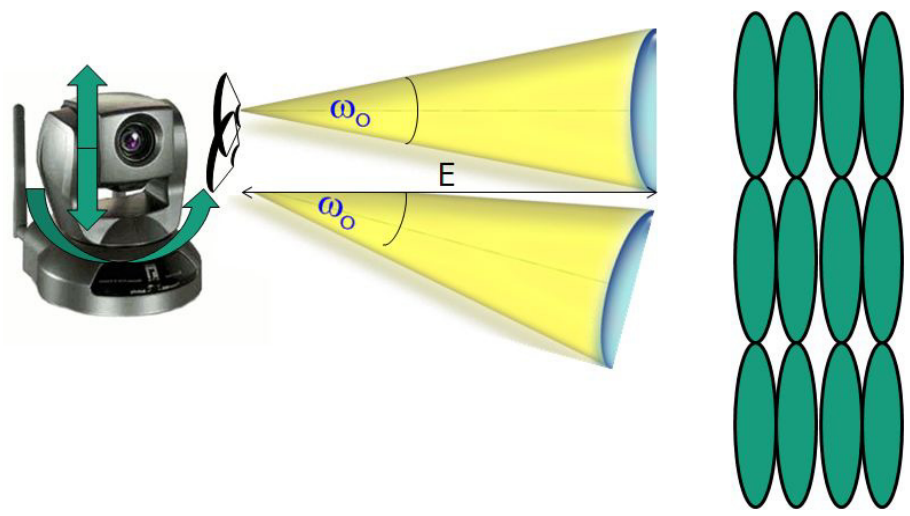

Figure 9: Scanning mechanism and illustration of footprints on the ground in distance E.

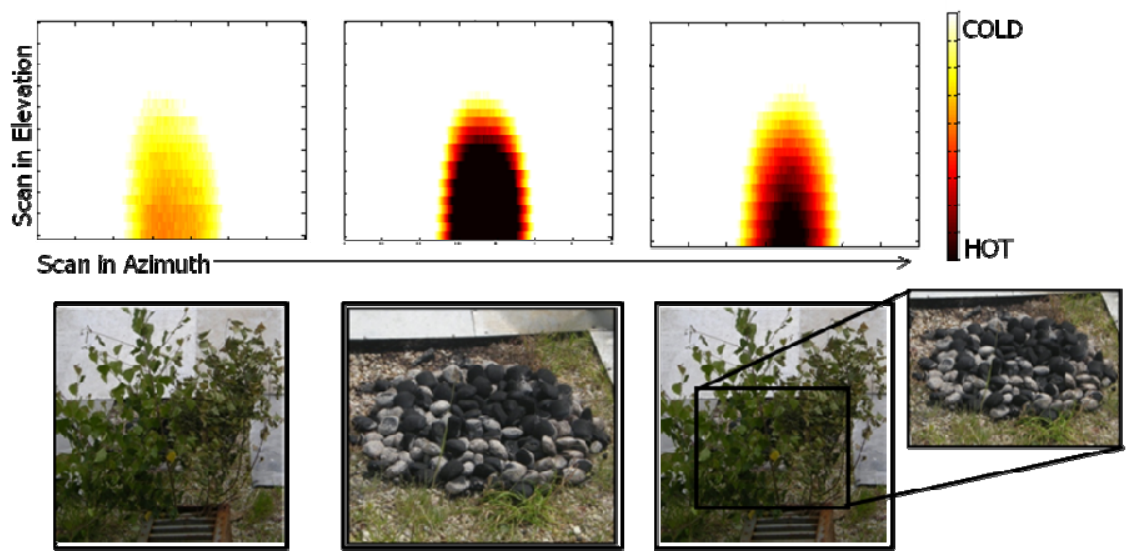

Figure 10: Radiometric measurement; left: tree alone, centre: charcoal alone, right: tree in front of charcoal fire.

The results of a $2 \mathrm{D}$ scanning radiometer and the good transmission characteristics of microwave radiation are demonstrated in fig. 10 by means of the transmission through a small tree. The radiometer was placed on a turntable to receive $2 \mathrm{D}$ images. The image to the left shows a scan of the tree alone, the image in the middle the radiation of a charcoal fire. On the right-hand side, the tree was placed in front of the fire. It can be seen that the radiation of the fire is attenuated in the tree but that it can still be clearly identified.

\section{Outlook}

A good interrelation of a very early and reliable smoke detection of forest fires, remote sensing techniques, logistics and technical support, training of fire 
fighters by simulation and an adequate extinguishing and rescue system will reduce damage and smoke impact on humans. The main focus has to be on early smoke detection because large and high-intensity forest fires are widely uncontrollable and cause very high risks.

To reduce false alarms of video-based systems, especially in hardly accessible terrain, a remote controlled UAV can fly to the place where a fire is assumed to confirm that the origin of the smoke is most likely a fire. After extinguishing a blimp can work as a fireguard.

The $22.3 \mathrm{GHz}$ radiometer is able to detect fire even under insufficient vision due to smoke emissions, dust or fog. Materials as leaves and thin walls can be partly transmitted by the microwave radiation. The current design can be further miniaturized using chip-based components.

Semiconductor gas sensors are widely immune against disturbances like steam, fog, dust pollution and condensing water. Together with an aspirating smoke detection system it is possible to detect even lowest smoke and gas concentrations to confirm an alarm under laboratory conditions as well as under outdoor conditions. Finally gas concentration, smoke density, results of radiometric measurement and pictures of a thermal imaging camera enable a good discrimination between alarm and false alarm.

\section{References}

[1] Henrichs, M., Armored and Tracked Vehicle for Rescue / Extinguish / Defend Missions, 14th International Conference on Automatic Fire Detection, AUBE '09, Duisburg, Germany, September 8-10, 2009.

[2] von Wahl, N., Heinen, S., Tobera, R., Nüßler, D., Brauns, R., Schröder, M., Knott, P., Krüll, W. \& Willms, I., Intermediate Report Internationale Waldbrandbekämpfung iWBB, FHR-Report Nr. 134, FGAN Research Institute for High Frequency Physics and Radar Techniques, Wachtberg, Germany, 2009.

[3] GTE, http://www.adicos.de

[4] Krüll, W., Willms, I., Tobera, R. \& Wiggerich, B., Early forest fire detection and suppression - an integrated approach, 14th International Conference on Automatic Fire Detection, AUBE '09, Duisburg, Germany, September 8-10, 2009.

[5] Tobera, R., Krüll, W. \& Willms, I., Optical smoke and gas sensors as an additional method for early wildfire verification, 14th International Conference on Automatic Fire Detection, AUBE '09, Duisburg, Germany, September 8-10, 2009.

[6] Hekatron, http://www.hekatron.de

[7] von Wahl, N., Heinen, S., Advantages of millimeter waves in fire detection and monitoring, 14th International Conference on Automatic Fire Detection, AUBE '09, Duisburg, Germany, September 8-10, 2009.

[8] Nüßler, D., Essen, H., von Wahl, N., Zimmermann, R. Rötzel, S., Willms, I., Millimeter Wave Propagation through dust, SPIE conference proceedings, September, 2008. 